\title{
Search for a Light Sterile Neutrino at Daya Bay
}

\author{
Hin Lok Henoch Wong*, on behalf of the Daya Bay Collaboration \\ Department of Physics, University of California, Berkeley, California 94720, USA \\ E-mail: henoch.wong@berkeley . edu
}

The Daya Bay reactor neutrino experiment's unique configuration of multiple baselines from six $2.9 \mathrm{GW}_{t h}$ nuclear reactors to eight antineutrino detectors deployed in two near (effective baselines $\sim 500 \mathrm{~m}$ and $\sim 600 \mathrm{~m})$ and one far $(\sim 1600 \mathrm{~m})$ underground experimental halls makes it possible to test for oscillations to a fourth (sterile) neutrino in the $10^{-3} \mathrm{eV}^{2}<\left|\Delta m_{41}^{2}\right|<0.3 \mathrm{eV}^{2}$ range. In this talk, I will present Daya Bay's latest results on the search for light sterile neutrino mixing. The relative spectral distortion due to the disappearance of electron antineutrinos was found to be consistent with that of the three-flavor oscillation model. The resulting limits on $\sin ^{2} 2 \theta_{14}$ constitute the world's best in most of the sub-eV mass region.

38th International Conference on High Energy Physics 3-10 August 2016

Chicago, USA

${ }^{*}$ Speaker. 


\section{Introduction}

The three-neutrino mixing framework, in which the flavor eigenstates $\left(v_{e}, v_{\mu}, v_{\tau}\right)$ mix with the mass eigenstates $\left(v_{1}, v_{2}, v_{3}\right)$ via the PMNS matrix has been extremely successful in explaining the results observed in most solar, atmospheric, reactor and long-baseline accelerator neutrino oscillation experiments. Despite this success, the hunt for the possible existence of additional neutrinos is actively pursued.

In the simplest extension of the Standard Model where only one sterile neutrino is considered in addition to the three active ones, if the neutrino mass is much smaller than its momentum, the probability that an $\bar{v}_{e}$ produced with energy $E$ is detected as an $\bar{v}_{e}$ after traveling a distance $L$ is given by

$$
P_{\bar{v}_{e} \rightarrow \bar{v}_{e}}=1-4 \sum_{i=1}^{3} \sum_{j>i}^{4}\left|U_{e i}\right|^{2}\left|U_{e j}\right|^{2} \sin ^{2} \Delta_{j i},
$$

where $U_{e i}$ is the element of the neutrino mixing matrix for the eigenstate $v_{e}$ and the mass eigenstate $v_{i}, \Delta_{j i}=1.267 \Delta m_{j i}^{2}\left(\mathrm{eV}^{2}\right) \frac{L(\mathrm{~m})}{E(\mathrm{MeV})}$ and $\Delta m_{j i}^{2}=m_{j}^{2}-m_{i}^{2}$ is the mass-squared difference between the mass eigenstates $v_{j}$ and $v_{i}$.

When $\left|\Delta m_{41}^{2}\right| \gg\left|\Delta m_{31}^{2}\right|$, the parameters $\Delta m_{41}^{2}, \Delta m_{42}^{2}$ and $\Delta m_{43}^{2}$ are virtually indistinguishable, and Eq. 1.1 can be approximated to

$$
P_{\bar{v}_{e} \rightarrow \bar{v}_{e}} \approx 1-\sin ^{2} 2 \theta_{14} \sin ^{2} \Delta_{41}-\sin ^{2} 2 \theta_{13} \sin ^{2} \Delta_{31}
$$

Thus, to first order, evidence for light sterile neutrino mixing consists of an additional spectral distortion with a frequency different from standard three-neutrino oscillations.

\section{Daya Bay Experiment}

The Daya Bay Reactor Neutrino Experiment is designed to precisely measure the neutrino mixing angle $\theta_{13}$, via the relative comparison of antineutrino rates and energy spectra at different baselines. Two near underground experimental halls (EH1 and EH2) and one far hall (EH3) houses a total of eight functionally identical antineutron detectors (ADs) in the configuration shown in Fig. 1. The results of this work is derived from the first 217 days of data acquired with six ADs deployed, and an additional 404 days with all eight ADs in operation.

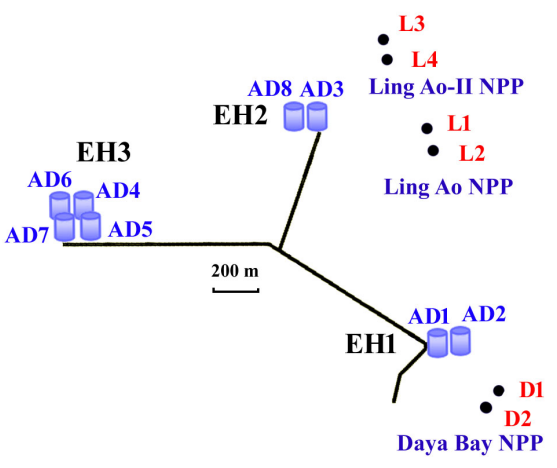

Figure 1: Layout of the Daya Bay experiment. The dots represent reactor cores, labeled as D1, D2, L1, L2, L3 and L4. The Daya Bay experiment started data-taking with six antineutrino detectors (AD1-AD6) installed in three experimental halls (EH1-EH3). In Oct 2012, two additional detectors (AD7 and AD8) were installed in $\mathrm{EH} 3$ and $\mathrm{EH} 2$, respectively. 
Reactor antineutrinos are detected via the inverse beta decay (IBD) reaction, $\bar{v}_{e}+p \rightarrow e^{+}+n$. The IBD candidates used for this light sterile neutrino search is identical to the data set that was used in Daya Bay's $\theta_{13}$ measurement [1]. A summary of the IBD candidates for the 6-AD and 8-AD periods, together with the estimated background levels and the baselines of the three experimental halls to each pair of reactor cores, is shown in Table 1.

Table 1: Summary of total number of IBD candidates and backgrounds, and baselines of the three experimental halls to the reactor pairs. Statistical and systematic errors are included.

\begin{tabular}{lccccccc}
\hline \multirow{2}{*}{ Site } & \multicolumn{2}{c}{ IBD candidates } & \multicolumn{2}{c}{ Backgrounds } & \multicolumn{3}{c}{ Mean Distance to Reactor Cores (m) } \\
\cline { 2 - 8 } & (6-AD) & $(8-\mathrm{AD})$ & $(6-\mathrm{AD})$ & $(8-\mathrm{AD})$ & Daya Bay & Ling Ao & Ling Ao-II \\
\hline EH1 & 205135 & 408678 & $4076.6 \pm 462.4$ & $7547.9 \pm 908.0$ & 365 & 860 & 1310 \\
EH2 & 93742 & 383402 & $1580.3 \pm 147.8$ & $5791.2 \pm 586.5$ & 1348 & 481 & 529 \\
EH3 & 41348 & 108907 & $1878.9 \pm 94.6$ & $2105.2 \pm 208.1$ & 1909 & 1537 & 1542 \\
\hline
\end{tabular}

The search for sterile neutrino mixing at Daya Bay is carried out through a relative comparison of the antineutrino rates and energy spectra at the three experimental halls. The unique configuration of multiple baselines to three pairs of nuclear reactors allows exploration of $\Delta m_{41}^{2}$ spanning more than three orders of magnitude. Fig. 2 shows the ratios of the observed prompt energy spectra at $\mathrm{EH} 2$ and $\mathrm{EH} 3$ to the best fit prediction from $\mathrm{EH} 1$ in the three-neutrino case. In this figure, the data are compared with the four-neutrino mixing scenario assuming $\sin ^{2} 2 \theta_{14}=0.05$ for two representative $\Delta m_{41}^{2}$ values, illustrating that the sensitivity at $\Delta m_{41}^{2}=4 \times 10^{-2}\left(4 \times 10^{-3}\right) \mathrm{eV}^{2}$ originates primarily from the relative spectral shape comparison between EH1 and EH2 (EH3).

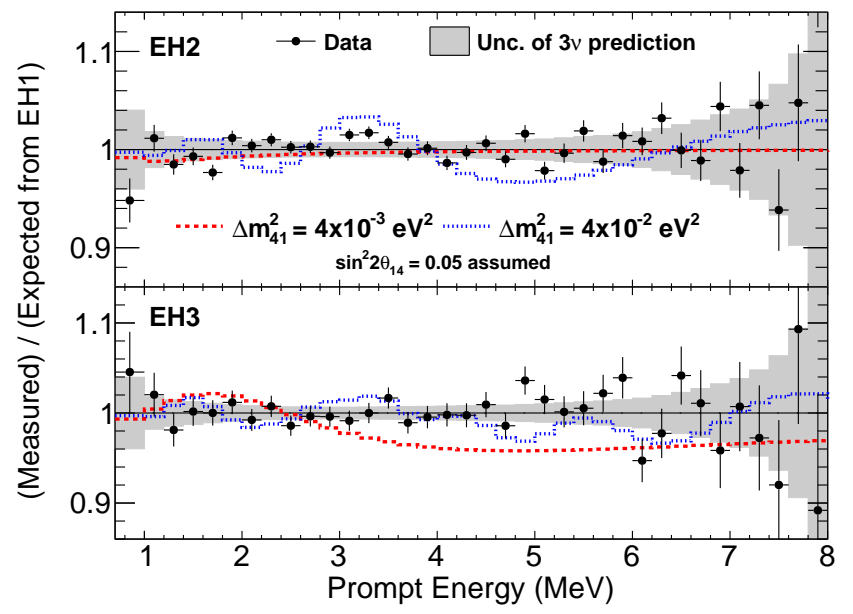

Figure 2: Prompt energy spectra observed at EH2 (top) and EH3 (bottom), divided by the prediction from EH1 with the three-neutrino best fit oscillation parameters from the Daya Bay analysis [1]. The gray band represents the one-standard-deviation uncertainty of the three-neutrino oscillation prediction. Predictions with $\sin ^{2} 2 \theta_{14}=0.05$ and two representative $\Delta m_{41}^{2}$ values are also shown as the dotted and dashed curves.

\section{Results}

The minimum $\chi^{2}$ value obtained with a free-floating $\Delta m_{41}^{2}, \sin ^{2} 2 \theta_{14}$ and $\sin ^{2} 2 \theta_{13}$ is $\chi_{4 v}^{2} / \mathrm{NDF}=$ 129.1/145, where NDF stands for the number of degrees of freedom. The corresponding value in the three-neutrino scenario, in which $\sin ^{2} 2 \theta_{13}$ is the only free parameter, is $\chi_{3 v}^{2} / \mathrm{NDF}=134.7 / 147$. The p-value of observing $\Delta \chi^{2}=\chi_{3 v}^{2}-\chi_{4 v}^{2}=5.6$ without sterile neutrino mixing is determined to be 0.41 using a large sample of Monte Carlo pseudo-experiments. No apparent signature for sterile neutrino mixing is observed. 
The limits in the $\left(\left|\Delta m_{41}^{2}\right|, \sin ^{2} 2 \theta_{14}\right)$ plane are set by two independent approaches, the first of which follows the Feldman-Cousins method [2], the second approach uses the $\mathrm{CL}_{\mathrm{s}}$ statistical method [3, 4]. Fig. 3 shows the $95 \%$ exclusion contours from both methods. These results set the most stringent limits to date on $\sin ^{2} 2 \theta_{14}$ in the $2 \times 10^{-4} \mathrm{eV}^{2} \lesssim\left|\Delta m_{41}^{2}\right| \lesssim 0.2 \mathrm{eV}^{2}$ region.

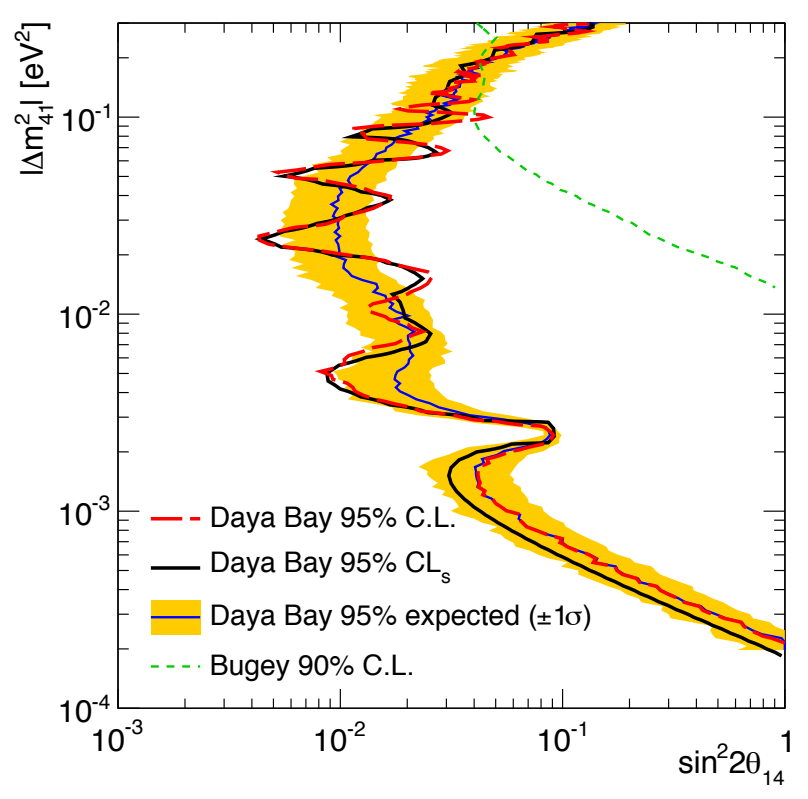

Figure 3: Exclusion contours in the $\left(\sin ^{2} 2 \theta_{14},\left|\Delta m_{41}^{2}\right|\right)$ plane, under the assumption of $\Delta m_{32}^{2}>0$ and $\Delta m_{41}^{2}>0$. The red long-dashed curve represents the $95 \%$ C.L. exclusion contour with the FeldmanCousins method [2]. The black solid curve represents the $95 \% \mathrm{CL}_{\mathrm{s}}$ exclusion contour [3]. The expected $95 \%$ C.L. $1 \sigma$ band in yellow is centered around the sensitivity curve, shown as a thin blue line. The region of parameter space to the right side of the contours is excluded. For comparison, Bugey's [5] 90\% C.L. limit on $\bar{v}_{e}$ disappearance is also shown as the green dashed curve.

\section{References}

[1] F.P.An et al. (Daya Bay Collaboration), New Measurement of Antineutrino Oscillation with the Full Detector Configuration at Daya Bay, Phys. Rev. Lett. 115111802 (2015) [hep-ex/1505. 03456 ]

[2] G.J.Feldman and R.D.Cousins, Unified approach to the classical statistical analysis of small signals, Phys. Rev. D 573873 (1998) [physics.data-an/9711021]

[3] A.L.Read, Presentation of search results: the $C L_{s}$ technique, J. Phys. G 282693 (2002)

[4] X.Qian, A.Tan, J.J.Ling, Y.Nakajima, and C.Zhang, The Gaussian $C L_{s}$ method for searches of new physics, Nucl. Instrum. Meth. A 82763 (2016) [hep-ex/1407. 5052]

[5] Y.Declais et al. (Bugey Collaboration), Search for neutrino oscillations at 15, 40 and 95 meters from a nuclear power reactor at Bugey, Nucl. Phys. B 434503 (1995) 